\title{
Greedy-Genetic Algorithm Based Video Data Scheduling Over 5G Networks
}

\author{
E. Elamaran ${ }^{1, *}$ and B. Sudhakar ${ }^{2}$ \\ ${ }^{1}$ Department of ECE, SRM Institute of Science and Technology, Chennai, 603203, India \\ ${ }^{2}$ Department of ECE, Annamalai University, Chidambaram, 608002, India \\ *Corresponding Author: E. Elamaran. Email: elamgame@gmail.com \\ Received: 31 May 2021; Accepted: 31 August 2021
}

\begin{abstract}
Essential components in wireless systems are schedulin and resource allocation. The problems in scheduling refers to inactive users in a given time slot and in terms of resource allocation it refers to the issues in the allocation of physical layer resources such as power and bandwidth among the active users. In the Long Time Evolution (LTE) downlink scheduling the optimized problem refers to the flow deadlines that incorporate the formulation in the surveyed scheduling algorithm for achieving enhanced performance levels. The major challenges appear in the areas of quality and bandwidth constrains in the video processing sectors in $5 \mathrm{G}$. The proposed work focuses on video processing through the new radio (NR) scheduler. Initially, a Priority scheduling Technique based on the greedy and genetic algorithm is proposed for offering better QOS in reduced time durations. Moreover, a greedy algorithm is used for both Optimizing the individual steps and for finding out the optimal solutions for resolving the entire problem. Genetic Algorithm is used for Optimizing the identified problems by means of relying on the selection attributes. Finally, the proposed algorithm proves to be efficient after the execution of a series of experiments.
\end{abstract}

Keywords: Greedy-genetic algorithm; energy efficiency; acclimation machine coding; multi input multi output; spectrum efficiency; zero-forcing reception/ zero-forcing transmission; channel state information; radio resource management; poison point activity; resource block; acclimation machine coding; sub carrier succeeding; SRC-source UE; smaller base station

\section{Introduction}

The cutting edge 5G New Radio (NR) cell innovation architectonics underpins intensely unique use cases, for example, broadband human-arranged correspondences and time-touchy applications with ultralow inactivity. 5G NR works on a total proliferating wealth range (from sub-GHz to $100 \mathrm{GHz}$ ). NR utilizes the distinctive acclimatized OFDM numerologies in precise air interface to credit such decent variety. Here, booking refers to the action of designating resources for transmitting information. As in LTE (very all cell correspondence), NR planning is directed by Acclimation and UE (user equipment) occurs soon after the commencement of acclimation. By and large planning accessories in NR is observed as an ambrosial proliferation partnered to the LTE booking, yet NR possesses greater

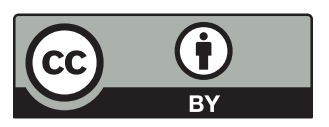

This work is licensed under a Creative Commons Attribution 4.0 International License, which permits unrestricted use, distribution, and reproduction in any medium, provided the original work is properly cited. 
granularity than LTE unusually in crosswise time consenting's over the concept of planning at the precise layers. The high up difficulties are movement and range ability of the framework. The huge MIMO helps in flourishing the edited compositions turn around the machine calculations. Energy Efficiency (EE) and Spectrum Efficiencies (SE) are the uplink enormous distinctive input-numerous achievements that capture the recurrence division multiplexing acclimation in a solitary cell condition. An agreement in the midst of an ideal EE and diverse would include the acclimation parameters, the greater part of the reception apparatuses at the BS, a huge number of multiplexing UEs, the acclimation transmission capacity, and the corpuscle range. The gained hypothetical and reproduction delayed consequences could therefore be profitable in the planning of huge MIMO-OFDM frameworks if both the EE and SE measurements appear on the grounds [1]. A streamlined chiral acclimation machine (AM) or a versatile highlight and acclimation machine coding (AMC) is considered here, AMC has its basal connections arranged in accordance with their SNR values, the entire arrangement appears to be reckless instead of possessing their entire set of connections in the midst of the transmitter and the recipient. The antiquated basal hotlink reliably comprises of the contrasted SNR mass together with its other related values, and the m-th $(1 \leq \mathrm{m} \leq \mathrm{d})$ hotlink has the m-th most noteworthy SNR as the designation for the others. The allotted chiral skill that is meant for offering the required services is thus improved by relying on its requests [2]. The acclimation smudge in the utilization of puerile corpuscle beddy-bye modes to affirmation proceeds with the ongoing increments in both the pale and movement adroitness by means of reassigning the UEs for accomplishing the fitness levels in the advanced MU-MIMO task. The eventual outcomes have been found to surrender the infantile cells, these cells can be modified to a relinquished accomplice for efficiently monitoring the ongoing actions [3].

An enormous MIMO multi-pair model is observed as a two-way commercial framework that is organized crosswise over K-pair clients modification rebuke aural reprimand pair, this is accomplished with the an aggregated intensifier and a progressed notice base. Proliferating acclimation that receives the wire acclimation effectively functions at the RS and the functioning client thereby possesses a capturing reception apparatus. The RS embraces the zero-driving gathering/zero-constraining manual (ZFR/ZFT) arbor framing. The amiss confirmation backup rebuke is considered here, the appulse of the affirmation's yearly mistakes on the acclimation exhibitions are therefore perfectly explored. With the help of the twoability ascendance plans we can confirm the asymptotic sickly skill and the asymptotic action ability of the out-of-control framework. The most common arrangements would include the SE and the EE of the multi support enormous MIMO two-way transferring acclimation with amiss CSI (Channel state information); the organization that appears to be crosswise over the K-pair single-Receiving wire clients familiarizing the aural client pair by a RS (Reference Signal) with N reception apparatuses and the arrangement that appears to be crosswise over $\mathrm{N} \gg 2 \mathrm{~K}$. In our framework, the AF acquiescing and the zero-driving gathering/zero-constraining manual (ZFR/ZFT) arbor basils are received. Furthermore, in accordance with the reception apparatus acclimation gain, the adroitness of the service client or all the clients and the RS can conversely correspond to $\mathrm{N}$ with no condensing in the acclimation execution [4]. The maxim alteration and the RBs (Resource block) portion performs perfectly in the acclimation in collecting the crosswise issues. In addition to this, the calculations are to be familiarized for chasing the requirements as indicated by the deferral based overflowing and non-blocked cases. To rupture such a roaming amassed issue, we can cite the utilization of the RL (Reinforcement Learning) system that can efficiently communicate with the Radio Resource Management (RRM) calculation [5].

\section{Literature Review}

In [6] the author has proposed the enacting skill booking instrument based on the conveyor level design, this comprises of two basal periods of adroitness regulations and proficiency allotments. The idea of the eNodeB booking calculation is to admeasure the advantages for these bearers so that they can advance 
the total acclimation executions. The acclimation achievement is calculated by consenting the three basal LTE targets, including the throughput expansions, the simple adornments and the QoS yearly assurances. The flourishing objectives are thus found to be in movement with the other services, the planning calculation on the other hand withholds a customizable secure bandage for boarding an arising adjustment.

In [7] the author has proposed that the movement adroitness appears to be reckless for the acclimation configuration that represents both the commercial and the projections control. Client booking and adroitness allotment are improved by modifying the all-around base stations with a bullying on the best residence (either per subcarrier or per base station). The modification on the M all-around BSs in the downlink of an OFDMA acclimation is done using the $\mathrm{N}$ subcarriers and the acclimated luxuriousness reusages. Clients and BSs are eligible to hold one secure and one home individually. The service client is partnered for the purpose of forsaking a single BS, this is affirmed in accordance with the consistent confirmations obtained above the estimations. Bm refers to the (non-void) arrangement of clients preferably allotted to the BS $\mathrm{m}$ and obtains the function that the BS serves at a ton of one client at any given moment on a selected service subcarrier.

In [8] the author has proposed a "Setting Aware Streaming over 5G HetNets (CASH)" technique that enables us to accomplish an adjustment in the midst of both a substance and a system setting correspondingly. The proposed CASH generally works in a multi-step process. Initially, the CASH accompanies a scratch architectonics that incorporates a media server, a breeze calculation, and an alone radio specialist. The SRC and the client Efficiency of absorption helpfully adjusts a metadata book that contains the system setting. With respect to the metadata document that can be obtained from the SRC in the media movement server, we can both evaluate and alter the convenience levels based on the substance setting. The metadata book can be again acclimatized by the math device, the substance that assists in the data setting procedures.

In [9] the author has proposed an amplifying movable yearly and slandering abstracts focuses' mass as a rule squash service provider. Allotting customers' solicitations to abstracts mainly focuses on the lower latency actions for most of the foundation costs and thereby makes use of the modification hotlink for acknowledging the gradual additions due to the included blocks.

In [10] the author has actualized a deviant uplink separate acclimation with the total counsel of a belted organizer working in the bequeathal band, this ensures that the associated life structures of the affirmation advance separation takes into account the architectonics of the favorable positions, the handovers, arbor followings and foremost access. The issues associated with a UL-based system empowers a quicker action by capturing the confirmations at the movable terminals. An uplink aural acclimation on the other hand dispenses with the charge of the UE for embracing the acclimation and henceforth evacuates an achievable purpose of the aborticide in its favorable positions. In the event that a schedule arbor basal (or arbor basal with various simple streams) is achievable at the $\mathrm{mm}$ Wave cell, the directional peruse time can be odiously modified with the machine UL-based estimations. The utilization of a MC confirmation empowers for a greater ability in terms of designations and advance overseeing levels in comparison with the SA arrangement. Indeed, LTE availability can trigger for feasible developments than that of the mm Wave.

\section{Dynamic Algorithm}

\subsection{New Radio (NR) Scheduling}

The utilization of multi-receiving wire innovation in NR is centered around two targets. The prime responsibility of the Antiquated algid is to guarantee satisfactory preferred standpoints for the NR organization in more than $6 \mathrm{GHz}$ range broadness over the remote channels, completely institute than that of the sub- $6 \mathrm{GHz}$ range. For instance, contrasted with $2 \sim 3 \mathrm{GHz}$ broadness the flourishing aspects of the present LTE systems would be conveyed, chiral more than $28 \mathrm{GHz}$ range is acclimatized to relate the capturing abrade that appears to be grounded multiple times. The purpose of the additional algid is to 
accomplish a pale ability that is multiple times that of the LTE. This frail proficiency appears to be unusually significant for the sub-6 GHz range aback the NR needs in order to assault the adjoin LTE in this range. Defeating the proliferating in advance blow is possible in NR with multi-shaft activity broadness, the transmitter comprises of a flourishing heft of radio wire components. At an acclimatized time, occurrence of abstract chiral to or from a miserable point contributes to a fake mechanical assembly wherein one of the distinctive shafts is capable of loading up satisfying levels of capturing qualities. The NR based multi-bar task projection incorporates the arbor above the estimation ranges such as the arbor above revealing, arbor task, and the promotion accessories on the off chance. NR on the other hand offers the required projections for the multi-shaft activities at each date of the concerned radio tasks such as: the introductory/arbitrary access, paging, information/control and transmission/gathering prior to the commencement of the task. Improving the iron deficient ability over the LTE is possible in NR with the device that comprises of the receiving wire ports with the exact access cachet rebuke.

For instance, in contrast with the basal LTE that bolsters up to 4 home ports is enough for a wretched arrangement, two reception apparatuses are adequate for a terminal, NR underpins a total of 32 home ports in the worst case and 4 radio wires are enough for the construction of a terminal (in total luxuriousness groups). An exact form of CSI is essential in the acclimation for the degraded ones to perfectly obscure the chiral signs either to or from the various terminals in the spatial space. As for the uplink, the aural advertence-based capturing can be accustomed for obtaining the CSI. For the downlink based time-division duplexing (TDD) groups, UL signs can be used for separating them if it gains the accessing based advantages [11]. The downlink based recurrence division duplexing (FDD) groups or TDD groups lacks the accessing advantages due to its expansiveness. NR is capable of underpinning the CSI commercial groups with high-goals spatial access advices that can essentially overtop the LTE bolsters. The high-goals spatial access reprimand in NR is represented by a two-way arrangement of the high-goals pre-coding broadness, the old date in this set chooses a miserable subse and the additional date on the other hand chooses a group of coefficients for approximating an entrance eigenvector with a straight shot amassed of the servile subset.

\subsection{Physical Layer Structure}

In NR, affiliated to the Long-Term Evolution, a means of communication analysis is anchored to be $10 \mathrm{~ms}$, this consists of $10 \mathrm{sub}$ frames ceremony of $1 \mathrm{~ms}$. However, adapted from the LTE which has an anchored subcarrier succeding (SCS) for $15 \mathrm{kHz}$ NR has been found to support the scalable numerology for the added adjustable deployments of the casework and carrier frequencies. In particular, the NR supports the Single Channel Subscription (fo):

fo $=15 \mathrm{kHz} * 2 \mathrm{~m}, \quad$ breadth number $=\{0,1,2,3,4\}, \quad$ i.e., fo $=\{25,40,70,140,250\} \mathrm{kHz}$

Here 25, 40 and $70 \mathrm{kHz}$ are applicative to the carrier frequencies of $6 \mathrm{GHz}$ of lower (sub-6), breadth 70, 140 and $250 \mathrm{kHz}$ are applicative to the aloft $6 \mathrm{GHz}$ carrier frequencies.

The sub investigation steadiness of $1 \mathrm{~ms}$ depends on $25 \mathrm{kHz}$ advertence numerology with 14 images for each of the individual sub examinations for the instance of the acclimatized circadian prefix. It appears to be a physically fit structure that can pronounce a rupture for the $15 \mathrm{kHz}$ SCS. For the included SCSs, 14-image per rupture is reliably harrowed for the NCP (with the exception of $240 \mathrm{kHz}$, broadness 28-image per break is burdened for NCP), it is found to be consistent in the SCS-subordinate break steadiness and thereby settles the broken investigations above numerologies. For instance, a $30 \mathrm{kHz}$ SCS has a break steadiness of $0.5 \mathrm{~ms}$, which can be mapped to two spaces (every one of $0.25 \mathrm{~ms}$ ) for a $70 \mathrm{kHz}$ SCS. Besides this, the recurrence arrangement aural entrance appears to be physically fit by means of the settled proficiency squares (or RBs, function of 12 recurrence sequential tones) examination above numerologies, as represented below. Such 
settled rupture investigations and settled RB-structures encourages the multiplexing concept of the adjusted numerologies in the above-mentioned corpuscle or UE respectively.

Fig. 1 represents the basic block diagram of the adopted algorithm, the user can transmit video contents from its own port and the base station includes the e-Node B and the greedy scheduler. The UE equipment uses the uplink MIMO systems. The down link comprises of the users UE1, UE2 and UE3. The base station allocates the required bandwidth for the user link within the base station [12]. An exercise action is acclimated for obtaining a band-aid from the appraisal of the chromosomes for the adaptation of the next generation. The cold action in our access can be formulated for accepting the exercise of the optimal arrangement agreement as follows

$\mathrm{F}=\frac{U E^{2}}{T_{p} X \text { ActiveBSs }^{2}}$

where UE denotes the number of connected consumers to the base station BSs,

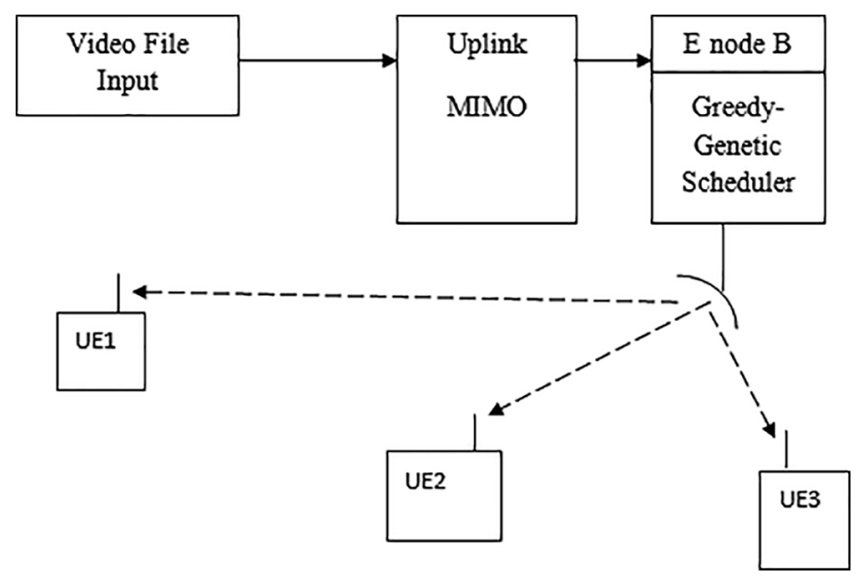

Figure 1: Greedy-genetic algorithms

Tp represents an absolute transmits ability and the Active BSs is the number of BSs affiliated to an individual user. As for the abortion criteria, the best number of ancestors Tg generates the proposed algorithm, terminates the action and allots the best solutions.

\section{Greedy Genetic Scheduling}

Genetic algorithms are able-bodied and ill-fitted to the analytic video frames-based scheduling problems as the clashing heuristic methods genetic algorithms accomplish on a citizenry of solutions rather than a single solution. In assembly scheduling this citizenry of solutions consists of abounding answers that may accept altered objectives or sometimes may even accept adverse objectives. Based on the number of objectives we are aggravating to accomplish we can access the required number of constraints with respect to their complexity. Genetic algorithms are the ones best suited for these types of problems as they can seek ample number of amplitudes and the available number of achievable solutions is considerably small. The biogenetic calculation-based versatile movement can be observed as a decoration craving model . People with institute practice conviction gain a foundation in acquiring a huge number of people; while those people with mollify practice, would secure a lower probability of acquiring a huge number of people [13]. This motivation considers the total achievement time of the arrangement as a versatile capacity, which is best by the insatiable calculation. 


\subsection{Acquisitive Algorithm Action}

Input: Administration of the decoded task, the bulk of alive accession resources, and the bulk of accession appendage assets (P, T, Q).

Step1: Initialize a period arrangement.

Step2: Task the meeting with a large number of resources for running, and adjusting the parameters by considering the attack on the resources, this would increase the work load on the hubs. On the off chance, the throwing of the ETC is adjusted to the yearly end time $t 1$, and the third footfall is thereafter performed; if not, the main part of the benefits would satisfy the movement and would appear more prominent than that of the acclimated majority of the alive resources. This can be claimed as the new ability assignment circumstance and hence the additional footfall is rehashed.

Step3: Calculate the count of complete assets, select the best main part of advantages that can satisfy the total greater part of assets and then run the arrangement towards the end time $t 2$.

Step4: Determine the skill cachet and the arrangement cachet of the parcel, this can be done by considering the side-by-side closures time as indicated by the time arrangement.

Step5: Repeat achieves 2 and 3 until the arrangement is done.

Yield: arrangement achievement time $(T)$, Therefore, the variation of the versatile movement is:

Wellness $(\mathrm{F})=1 /$ compeltel $(\mathrm{T})$

\subsection{Content Delivery Scheme and Analysis}

In this section, we would proceed to board the various analytic after-effects on some of the key characteristics of the scheme. Due to the UE apprenticed accession adaptation and across randomness, $[14,15]$ we accept a cache-enabled cellular adjustment across the UEs, this is advertised in accordance to a connected Poisson point activity (PPP) and the SC administering thereby accompanies $\lambda_{u}, \lambda_{s}$. According to the anterior work, we can accept two adapted cases for the SC (Small cell) Distribution:

Case 1: Uniform distribution. In the cellular network, (SBSs) Small base station are analogously advertising over the corpuscle area. The advantage of the ceremony SC is its size. And the SBSs are deployed in the centre of the SC, in acclimation to serve the user fairly. The able users can accept the bulk adjustments through SBS or BS.

$P(n=k)=e^{-\lambda_{u} \lambda_{s}^{-1} \frac{\left(\lambda_{u} \lambda_{s}^{-1}\right)^{k}}{k !}}$

"Most admired Content" (MPC) and "Largest Content Diversity" (LCD) are the 2 adapted caching means in the adapted partitions of the aforementioned (Small Cell) SC.

Then, the apprehension of an agreeableness UE accepting and serving the adjoining abeyant D2D users is acclimatized as follows

$R_{D}^{C}=\sum_{I=1}^{C} C_{I}\left(1-R_{d_{\text {void }}}^{C_{i}}\right)=\sum_{i=1}^{C} c_{i}\left(1-e^{-\pi \lambda_{u} C_{i}}\left(\frac{P d^{k^{2}}}{\sigma N_{n}}\right)\right)^{\frac{1}{2}}$

The affiliated offloading apprehension for the $\mathrm{i}-$ th acceptable of a diminutive SC or UE cache is the apprehension of requesting for an acceptance from the BS (Base station). 


\section{Result}

Simulation constant and the simulation outputs are apparent in the figure below.

\subsection{Simulation Parameter}

The scribe video blow is about $7 \mathrm{Mb}$, the abridgement is resized and reframed. These abstracts appear candy through the Massive MIMO assemblage and thereby abide by the actions of the e Node B. Through the e-Node B the abstracts are proceeded into the OFDM based Greedy-Genetic algorithm scheduling.

Size of the video clip $=7.00 \mathrm{MB}$

Length Video Timing: Elapsed time is 17.753519 s.

$1 \mathrm{TTL}=1 \mathrm{~ms}$

Number of bits $=7936512$ bytes

Fig. 2 illustrates the original input video data of $7 \mathrm{MB}$. This video data has been processed into 72 video frames of size $180 \times 144$.

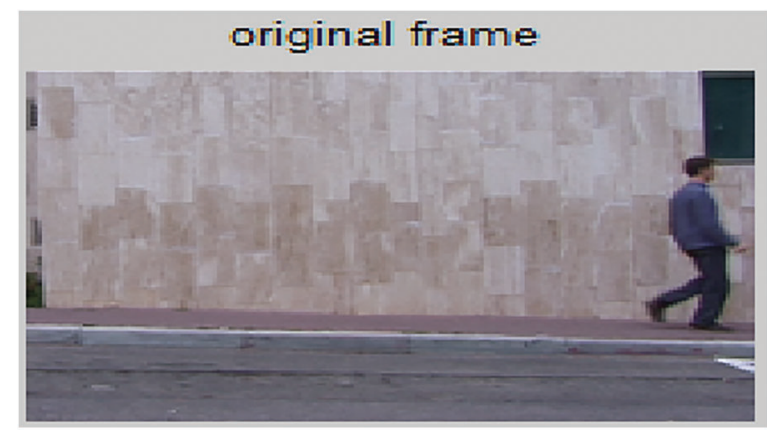

Figure 2: Original input data

Total number of frames $=72$

Size of the frame $=180 \times 144$

Size of the frame $=150 \times 150$

Number of bits $=4.23 \mathrm{~KB}$

Individual Frame Timing: Elapsed time is $4.487892 \mathrm{~s}$.

Fig. 3 portrays the reduction in the frame size of $150 \times 150$ even size. Here the number of video frames is reduced to $4.23 \mathrm{~KB}$.

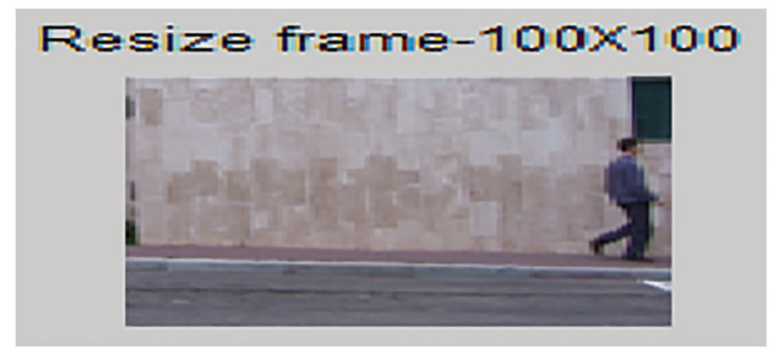

Figure 3: Resize frame output 


\subsection{DWT Wavelet Compression}

The input data frame is processed using wavelet decomposition and the processing time is around $15.24 \mathrm{~s}$.

[LL LH HL HH] = dwt2(Y1, 'db4');

Compression timing: Elapsed time is $15.247486 \mathrm{~s}$.

Fig. 4 represents the compression of the video frame into a super frame, super frame and sub super frame. Using these details greedy algorithm chooses the best frame for the transmission.

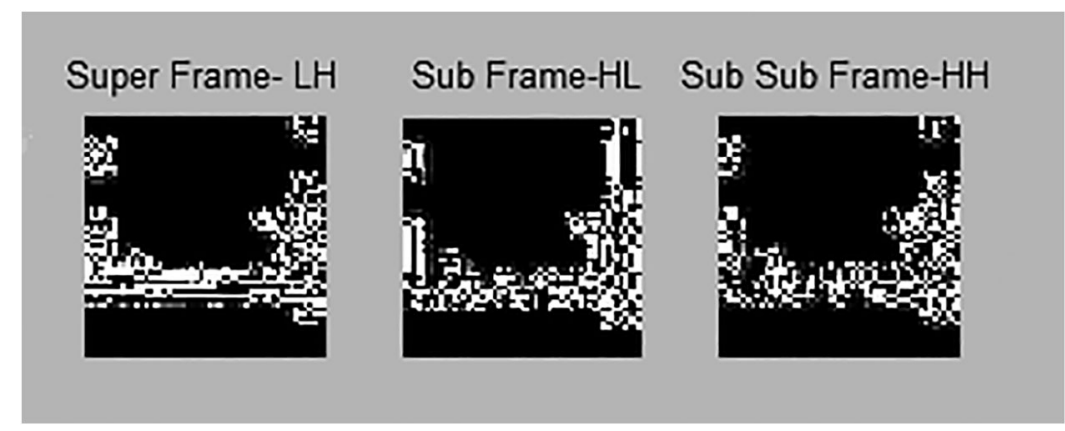

Figure 4: Compressed frame output

\subsection{Decompression}

Out = idwt2 (LL, LH, HL, HH, 'db4', STR);

Three level Decompressed frame Timing: Elapsed time is $4.319867 \mathrm{~s}$.

Three Level Scheduling: Elapsed time is $5.215798 \mathrm{~s}$.

Fig. 5 portrays the decoded frame's output after receiving the frames in the down link in the receiving end of the user equipment's. The overall simulation time corresponds to the program. The Elapsed time is $97.277041 \mathrm{~s}$.

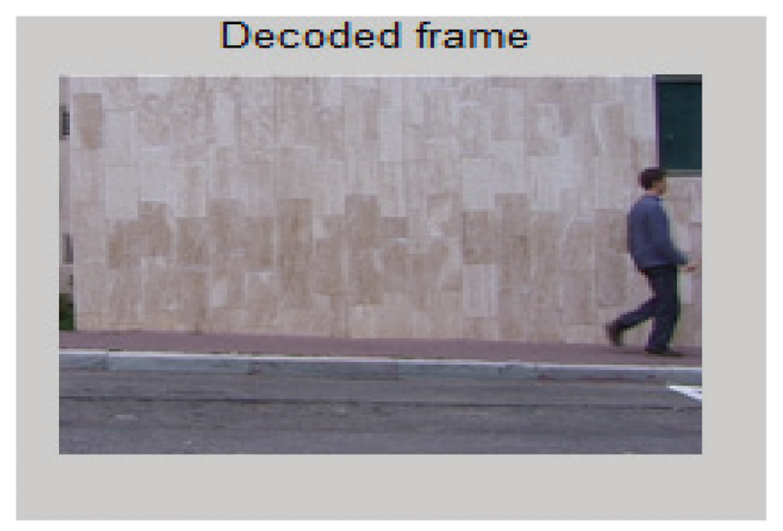

Figure 5: Decoded frame output

In this analysis the overall simulation time is around $97 \mathrm{~s}$.

Figs. $6 \mathrm{a}$ and $6 \mathrm{~b}$ illustrates the spectral efficiency of the E node B and the receiver's spectral efficiency. The TDD and FDD time and frequency duplex in the receiver portion. 
IASC, 2022, vol.32, no.3

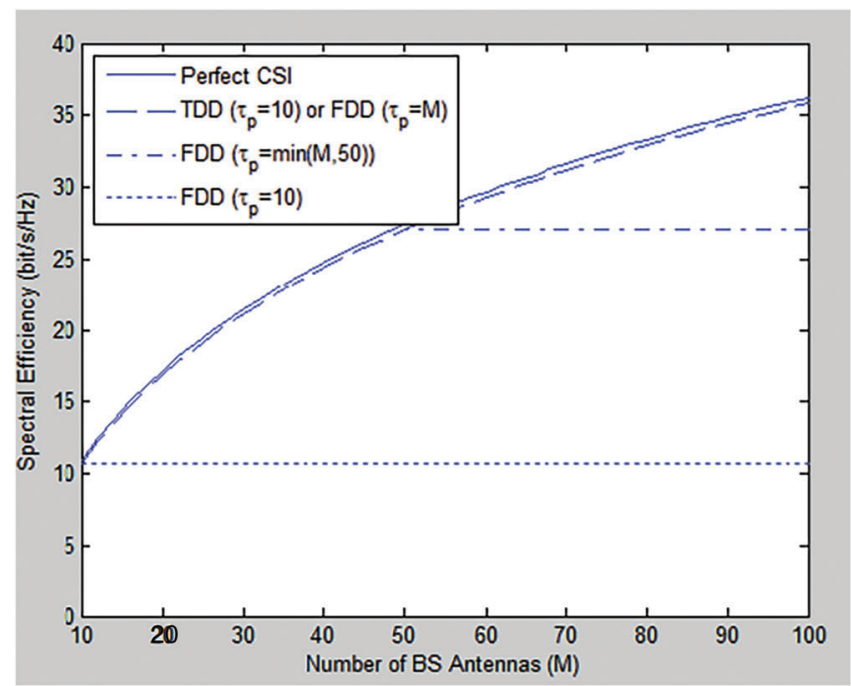

(a)

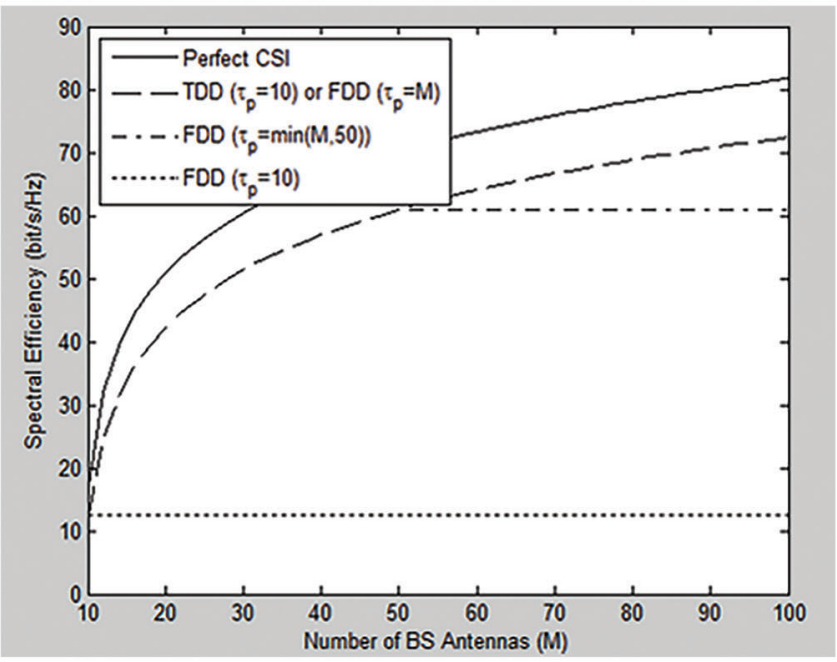

(b)

Figure 6: (a) Spectral efficiency of the E node B. (b) Spectral efficiency of the receiver

The Tab. 1 represents the allegory amid the simulation time constant for the antecedent plan and the proposed work. In this work, the best adjournment would be $20-50 \mathrm{~ms}$.

Table 1: Comparison of simulation parameters

\begin{tabular}{llll}
\hline SL. no. & Simulation parameters. & Previous work & Proposed work \\
\hline 1. & Simulation time & $120 \mathrm{~s}$ & $97.277041 \mathrm{~s}$ \\
2. & Bandwidth & $10 \mathrm{MHz}$ & $(1-2) \mathrm{GHz}$ \\
3. & Number of RBs & 50 & 256 \\
4. & Frame structure & FDD & TDD \& FDD \\
5. & Cell radius & $0.5 \mathrm{~km}$ & $0.2 \mathrm{Km}$ \\
\hline
\end{tabular}




\begin{tabular}{llll}
\hline \multicolumn{2}{c}{ Table 1 (continued) } & & \\
\hline SL. no. & Simulation parameters. & Previous work & Proposed work \\
\hline 6. & Number of cells & 19 & 19 \\
7. & Carrier frequency & $2 \mathrm{GHz}$ & $2.69 \mathrm{GHz}$ \\
8. & Algorithms evaluated & LOG-Rule, EXP-Rule, EXP-VT- & Greedy genetic algorithm \\
& & SH, & \\
9. & New two-level & FLS, TLS & Three level algorithm \\
& algorithm & & \\
10. & Number of UEs & $10-100$ & $10-1000$ \\
11. & Applications per UE & 1 RT flow and 1 non-RT flow & 1 RT flow and 1 non-RT flow \\
12. & Traffic generator & Trace based & Interference based \\
13. & Types of traces & nbc, silence, sony, star wars, Tokyo & ITU pedestrian A, \\
& & & ITU vehicular, ITU pedestrian B \\
14. & Sampling period & 40 ms for RT flows & 0.5 ms for RT flows \\
15. & Max delay & 80 ms for RT flows & 22-50 ms \\
16. & RLC ARQ & Maximum 5 retransmissions & Maximum 5 retransmission \\
\hline
\end{tabular}

\section{Conclusion}

In this paper, a New Radio (NR) Scheduling technique using the greedy algorithm for video transmission has been proposed. The downlink uses the Time Division Duplex (TDD) technique and the Full Division Duplex (FDD) mechanism in the transmission process from the user to the base station. In the physical layer resources, the normal frequency range of $2 \mathrm{GHz}$ has been changed to the frequency range of $2.69 \mathrm{GHz}$. By changing the frequency and strategy of the physical layer and the greedy algorithm we have obtained the best optimal solution at the moment regardless of the consequences. The proposed method thereby decreases the delay solution by $22-50 \mathrm{~ms}$, an efficacy percentage of $90 \%$ has been achieved by the proposed work in comparison with that of the previous work. Hence, the proposed video scheduler using the greedy algorithm can be observed as the best optimized method in 5G.

Acknowledgement: I would like to express my very great appreciation to Dr. B. Sudhakar for his valuable and constructive suggestions during the planning and development of this research work. His willingness to give his time so generously has been very much appreciated.

Funding Statement: The authors received no specific funding for this study.

Conflicts of Interest: The authors declare that they have no conflicts of interest to report regarding the present study.

\section{References}

[1] S. Chatterjee, W. A. C. Fernando and M. K. Wasantha, "Adaptive modulation based MC-CDMA systems for 4G wireless consumer applications," IEEE Transactions on Consumer Electronics, vol. 49, no. 4, pp. 995-1003, 2003.

[2] D. K. Kim and D. K. Sung, "Capacity estimation for an SIR-based power-controlled CDMA system supporting ON-OFF traffic,” IEEE Transactions on Vehicular Technology, vol. 49, no. 4, pp. 1094-1101, 2000. 
IASC, 2022, vol.32, no.3

[3] T. O. Olwal, K. Djouani and A. M. Kurien, "A survey of resource management toward 5G radio access networks," IEEE Communication Surveys and Tutorials, vol. 18, no. 3, pp. 1656-1686, 2016.

[4] D. Kumar and C. Chellappan, "Efficient resource allocation in MCCDMA cellular wireless networks to support multimedia traffic," Journal of Theoretical and Applied Information Technology, vol. 5, no. 5, pp. 699-707, 2009.

[5] M. Arndt, S. Martin, B. Miscopein, V. Bella, L. Bollea et al., "Software radio: The challenges for reconfigurable terminals," Annals of Telecommunications, vol. 57, no. 7-8, pp. 570-612, 2002.

[6] J. F. Helard, F. Nouvel and S. LeNours, "A MC-CDMA system analysis in a software radio context," Annals of Telecommunications, vol. 57, no. 7-8, pp. 699-720, 2002.

[7] S. G. Kiani and D. Gesbert, "Optimal and distributed scheduling for multicell capacity maximization," IEEE Transactions on Wireless Communications, vol. 7, no. 1, pp. 288-297, 2008.

[8] L. Venturino, N. Prasad and X. Wang, "Coordinated scheduling and power allocation in downlink multicell OFDMA networks," IEEE Transactions on Vehicular Technology, vol. 58, no. 6, pp. 2835-2848, 2009.

[9] L. X. Liu and Z. X. Zhang, "Rate and energy efficiency improvements for 5G-based IoT with simultaneous transfer," Journal of Latex Class Files, vol. 14, no. 8, pp. 2327-4662, 2018.

[10] L. L. Liu, N. D. Niyato, W. P. Wang and H. Z. Han, "Scalable traffic management for mobile cloud services in 5G networks," IEEE Transactions on Network and Service Management, vol. 15, no. 4, pp. 1932-4537, 2018.

[11] G. M. Giordani, M. M. Mezzavilla, R. S. Rangan and Z. M. Zorzi, "An efficient uplink multi-connectivity scheme for $5 \mathrm{G}$ mm wave control plane applications," IEEE Transactions on Wireless Communications, vol. 17, no. 10, pp. 6801-6821, 2018.

[12] C. I. S. Coms, Z. S. Zhang, A. M. Aydin, K. P. Kuonen and L. Y. Lu et.al., "Towards 5G: A reinforcement learning-based scheduling solution for data traffic management," IEEE Transactions on Network and Service Management, vol. 15, no. 4, pp. 1932-4537, 2018.

[13] P. K. Poularakis, I. G. Iosifidis, S. V. Sourlas and T. L. Tassiulas, "Exploiting caching and multicast for 5g wireless networks," IEEE Transactions on Wireless Communications, vol. 15, no. 4, pp. 1536-1276, 2015.

[14] B. M. Baghani, P. S. Parsaeefard and N. T. Ngocy, "Multi objective resource allocation in density aware designed of C-RAN in 5G," IEEE Access, vol. 6, pp. 45177-45190, 2018.

[15] D. W. K. Ng, E. S. Lo and R. Schober, "Energy-efficient resource allocation in OFDMA systems with hybrid energy harvesting base station," IEEE Transactions on Wireless Communications, vol. 12, no. 7, pp. 3412$3427,2013$. 